\title{
Using Morphological Operation and Watershed Techniques for Breast Cancer Detection
}

\author{
https://doi.org/10.3991/ijoe.v16i05.12999 \\ Sarah Faris Ameer ${ }^{(凶)}$, Zinah Tareq Nayyef, Zena Hussain Fahad \\ Dijlah University College, Baghdad, Iraq \\ sarah.faris@duc.edu.iq \\ Ibtihal Razaq Niama ALRubee \\ Wasit University, Al Kut, Wasit, Iraq
}

\begin{abstract}
Breast cancer is one of the leading causes of mortality between women, with one in eight women diagnosed with the disease, but early detection can reduce death rates. Therefore, continuous effort is being made to advance more effective methods for the early and effective diagnosis of breast cancer with high accuracy without human intervention. Classical attempts were manual, timeconsuming and ineffective in many situations. The purpose of this work is to detect and locate the presence of malignant tissues in the breast using the morphological technique in mammogram images to diagnose breast cancer because morphology is one of the most reliable methods for early detection of breast cancer. The proposed algorithm is developed using watershed segmentation after the preprocessing is completed by the median filter to eliminate any expected noise, and contouring the tumor by morphological techniques to take the best diagnostic for breast cancer in a mammogram image. Good results are obtained for the measurements used like MSE, PSNR, SNR, entropy for the mammogram images.
\end{abstract}

Keywords - Breast cancer, Mammogram images, Morphological operation, Watershed transforms, Image Processing.

\section{Introduction}

Cancer is a type of disease in which the cells are changed a propagate out of control. Cancer cells mass called tumor, which is name "tumor" after a part of the body where the tumor starts for the first time [1]. Breast cancer occurs when breast tissues change and increase rapidly without control, forming a mass of additional tissues as shown in Figure (1). These tumors can be either malignant or beginner [2]. The tumors in breast tissues can be distinguished by their shapes, as the benign tumors are round or oval, while malignant tumors are round and have a spiky outline that appears to be whiter than surrounding tissue due to a presence of calcium deposits which is known as calcification [3]. Breast cancer is the second the most hazardous cancer after lung cancer, with more than 11,000 women die around the world each year due to this disease [4]. Most studies have demonstrated that ladies with a family ancestry of breast cancer, 
regardless of whether the family ancestry of the mother or the father, are more likely to have the disease than men [5]. Primary prevention of this cancer is not possible because the underlying reasons for the disease are still unclear. Accordingly, the early identification of the disease is the basic method to promote diagnosis of breast cancer [6].

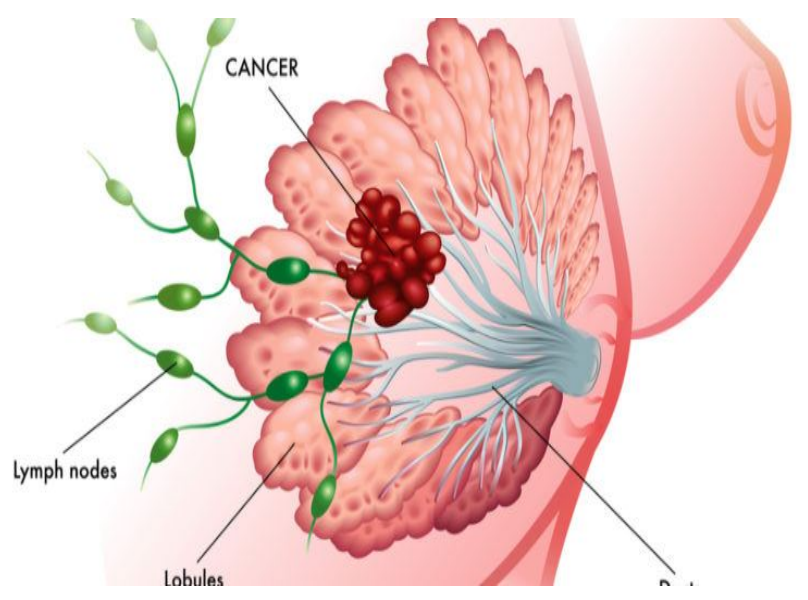

Fig. 1. Breast cancer

Three main methods of breast cancer screening are self-checking, clinical inspection, and mammography image. Mammography, also known as a mammogram, is a specific medical image using a low-dose X-ray system for breast screening and breast cancer diagnosis and is currently one of the best available ways for primary recognition of breast cancer [7, 8].

Image segmentation assumes a noteworthy role in a few handy applications, for example, medicinal science. Image segmentation means dividing images with different properties into regions that can be interpreted in a semantic manner so that the properties of each region can be defined and useful objects extracted $[9,10]$. In this paper, the image is segmented by relying on the concepts of watersheds transformation. Watershed is a powerful image segmentation tool to find regions that contain tumors in mammogram images and then perform morphological processes to more precisely recognize tumor boundaries and diagnose the tumor stage. The accuracy of the results derived from the algorithm used which has been determined based on the measurement standards for image errors.

\section{$2 \quad$ Related Work}

To our best of knowledge, the study by Samir M. Badawi, et al. (2017), the authors mainly focused on The double threshold was used to divide the mammogram, adding the boundary to the final image contour on the original image, which improved the detection and analysis of breast cancer easily through mammogram images [7]. 
In another study conducted by B. MONICA JENEFER and V. CYRILRAJ (2014), the authors focused primarily on enhancing the originality of the image to a higher and more logical level by eliminating noise and improving image quality by using noise removers with the EM algorithm respectively. The tumor region is then extracted by the modified watershed segmentation algorithm to be classified using SVM [6]. Another study by Jaya Sharma and Sujit Sharma (2011) suggested the abstraction of watershed lines from the topography of the input image. A slight modification was made to the method used to help reduce the number of areas obtained after fragmentation. A standard mammography database was used in the proposed method [11]. Ranjit Singh, et. al. (2009) They proposed a way to detect breast cancer by converting mammograms to a binary image. Then use some techniques to find the granular measurement of the tissue in the image without segmenting each and every object [4]. WenFeng et al. (2011) Proposed a different image segmentation method combining fuzzy C-Means (FCM) clustering technique and watershed algorithm. In this study yields a confirmed result in fragmentation compared to the conventional watershed algorithm by brain spectra and actual data [12]. In [13], the Authors established the prediction of diseases.

\section{Morphological Image Process}

It is a set of image processing strategies that bargain with the form of traits in an image. Typically, the morphological operation of the image is used to eliminate defects that are recognized during segmentation.

Mathematical morphology mainly depends on the relative order of the pixel, as well as a set of theories used to process and analyze images. Structural elements (SE) represented in the form of matrices that are used in the mathematical morphological process. $\mathrm{SE}$ is an image shape measurement feature that is used to perform different image processing operations. The morphological processing dependent on these two basic operations "Dilation" and erosion" [14] [15]:

I. In the morphological dilation, the object becomes larger or larger than its original size and its growth relies on the essence and shape of the structural element (SE). Morphological dilation process is used mainly to fill the gaps (missing pixels) in the continuous object because it adds pixels to the object boundary affects the intensity in this location and as a result blurring effect can be recognized.

II. The morphological erosion process is inverse of the dilation process. Erosion operation removes a layer of pixels from both the inner and outer boundaries of areas of the image, resulting in the loss of the object to its size or shrinkage. The noisy 'connection' among the two objects can be also eliminate using the morphological erosion.

III. The open morphological process is an erosion after dilation, and the close morphological method is performed by dilation after erosion. 


\section{$4 \quad$ Watershed Transforms}

The watershed is a methodology of alternative choice for image segmentation supported by the operation of mathematical morphology [12] [16][17]. Watersheds are one of the classified regions that rely mostly on segmentation. This segmentation methodology is wanted to reveal F [1some of the annoying and multiple parts of the images. The idea behind watersheds is directly taken from Earth science. When the terrain of the image is processed, the area is thought to be filled with a landscape or a flooded landscape by water. Watersheds can be the dividing lines for rain falling on the area. Another way is to assume that the landscape is immersed in a lake, with holes at perforated in native minima. The operation is turned off when the water level reaches the highest peak within the landscape. Therefore, the landscape is divided into areas or dishes parted by dams. These areas are defined as basins known as watershed lines that divide the input image into a group of areas [11].

Therefore, Watershed is considered a powerful means of segmentation because of its many benefits as being uncomplicated and fast, fully dividing the image and enabling complete detection of the boundaries of the objects in the image.

\section{Proposed Work}

Here, the authors explain the proposed algorithm utilized to diagnose breast cancer in which ultrasound images were used with a gray level value. different images with different stages of breast cancer examined by the algorithm and finally a diagnosis of the disease. 


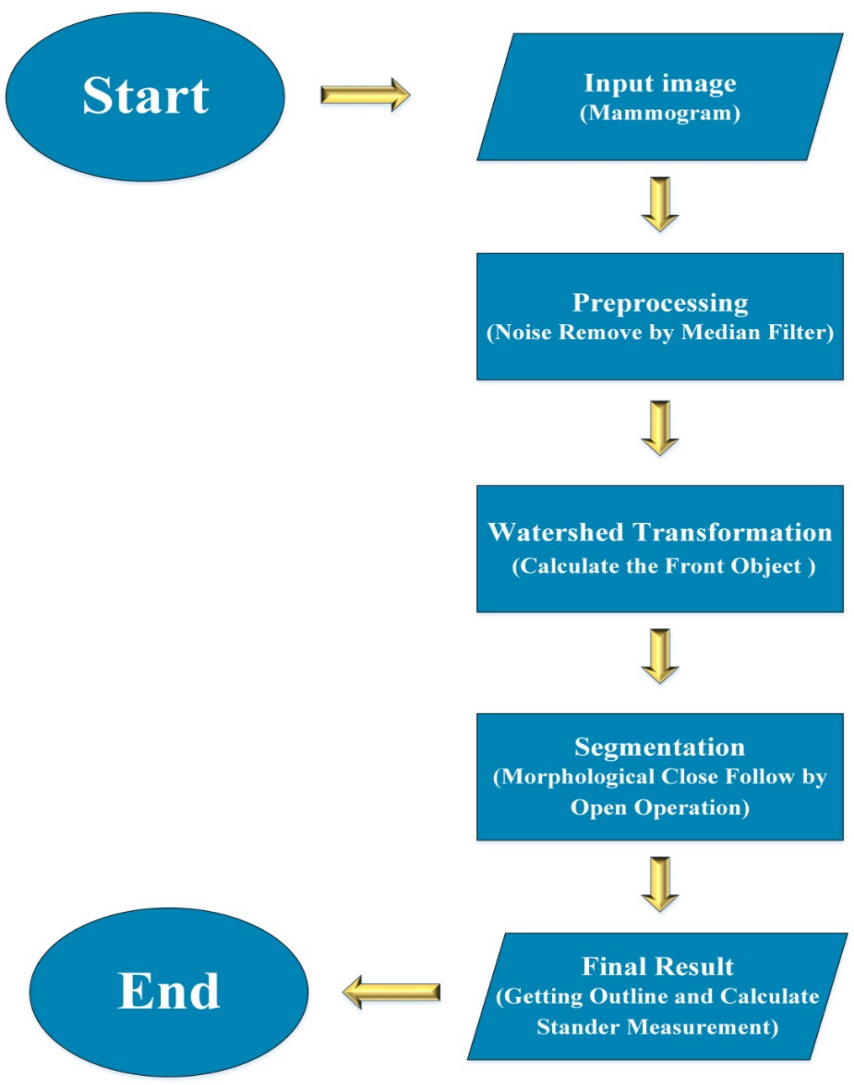

Fig. 2. Flowchart of Proposed Work.

\subsection{Input mammogram image}

A mammogram is medical imaging that helps in the early identification of breast cancer. The images size used in this paper $256 \times 256$ pixels. Figures (3), (4) and (5) show samples of the images used to determine the tumor in the proposed method.

\subsection{Image preprocessing}

In pre-processing, the input image is improved first by removing the noise to get an accurate result. The noise represents the unwanted area in the mammogram which is condensed by the median filter. The median filter better than a mean filter to eliminate the noise from the image without reducing the accuracy since it shows less sensitivity than the mean filter. 


\subsection{Watershed transform}

It Calculates a frontal object that connects pixels from within each of the foreground objects which is utilized for morphological operation open and close for reconstruction and refining the image.

\subsection{Segmentation}

The principal objective of the segmentation is to clarify or improve the image illustration to obtain a significant and more proper image in the analysis. In the mammogram image, the backdrop objects can appear more luminous, so the preparation stage is necessary to enhance image feature and generate segmentation results more precise. The first step in Segmentation is to convert the image into a binary image and extract the object into the image from the background using the threshold value (after many of the trials we have chosen the threshold value is 0.70 ), which removes any object connected to the border using the MATLAB imclearborder function, to finally settle of the object, to make the segmented object appear natural.

The final process that is used to detect breast cancer is a morphological process which uses the close process followed by an open process to extract the area that has a tumor of the image, then identifies the tumor area in the breast image and detects the stage of the tumor (If tumor is beginner, malignant, or normal).

\subsection{Result and discussion}

The proposed Approach gives a viable way to deal with breast cancer for the type of medical images namely mammography. The final images determine the accuracy of the algorithm applied in identifying areas containing tumors in the mammogram image, which help doctors detect breast cancer easily. Two types of segmentation algorithm used for identifying the tumor first by the watershed then followed the morphological operation, to obtain more accurate and error-free results. About 35 mammography images are examined to identify the tumor correctly. Figures below show the result of different Mammogram images with different stages of cancer, the performance of the suggested process is assessed using a set of evaluation metrics such as MSE, Entropy. PSNR, SNR which has shown good results. Table (1) illustrates some of the measurement results obtained from testing the proposed algorithm on a number of images. 

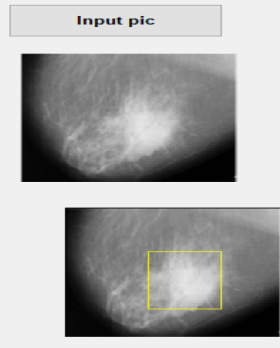

Getting outline
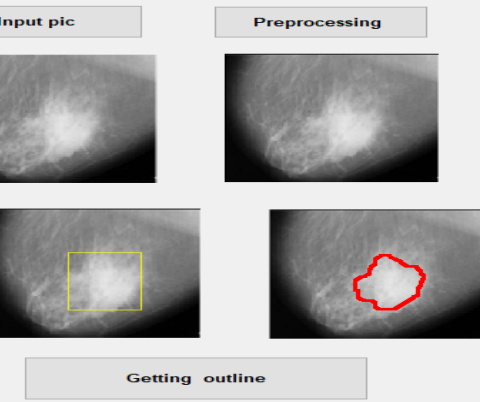

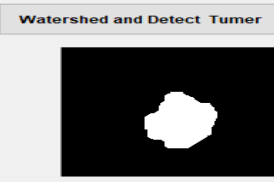

Stage of tumor Malign

MSE

SNR

PSNR

Entropy

Measurements

Fig. 3. Malign Breast cancer

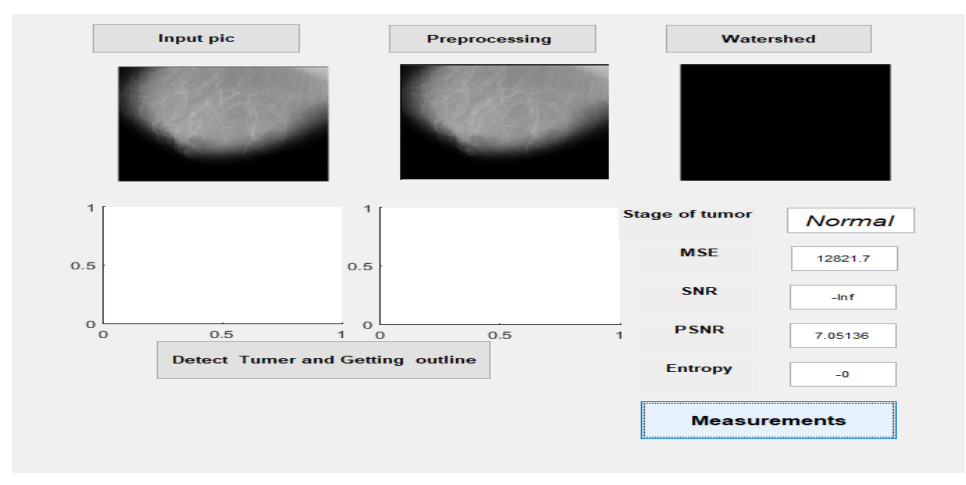

Fig. 4. Normal Breast

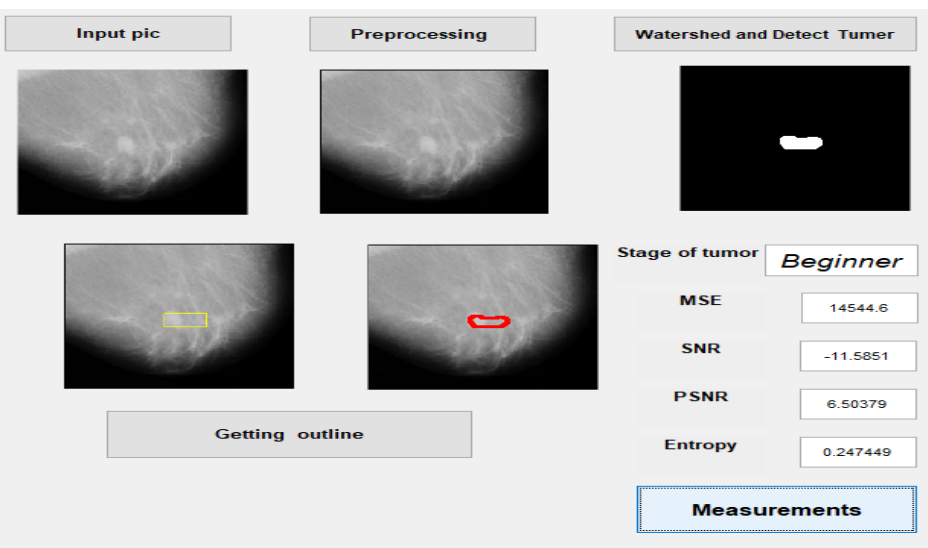

Fig. 5. Beginner Breast cancer 
Table 1. Measurements of different Mammogram images

\begin{tabular}{|l|c|c|c|}
\hline \multicolumn{1}{|c|}{ Type of image } & SNR & PSNR & Entropy \\
\hline Normal1 & - & 7.05 & 0 \\
\hline Normal2 & - & 5.75 & 0 \\
\hline Malign cancer1 & -2.602 & 7.19 & 0.813 \\
\hline Malign cancer2 & -3.63 & 6.163 & 0.8135 \\
\hline Beginner1 & -11.58 & 6.503 & 0.247 \\
\hline Beginner2 & -9.58 & 8.50 & 0.24 \\
\hline
\end{tabular}

The use of accuracy and sensitivity measures were a criterion for assessing the performance of the proposed approach and was calculated using the following equations:

$$
\begin{gathered}
\text { Sensitivity }=\mathrm{TP} /(\mathrm{TP}+\mathrm{FN}) \\
\text { Accuracy }=(\mathrm{TP}+\mathrm{TN}) / \mathrm{N}
\end{gathered}
$$

$\mathrm{TN}$ represents the number of real pros, false negatives $\mathrm{FN}$, false positives $\mathrm{FP}$, and $\mathrm{N}$ is the total number of images. In the proposed algorithm 35 images were used to test positive real images were obtained, 24 True Negative 9, False Positive 01 and False Negative 00, and then:

$$
\begin{aligned}
& \text { Sensitivity }(\%)=\mathrm{TP} /(\mathrm{TP}+\mathrm{FN}) \\
& =24 /(24 / 0)=100 \% \\
& \text { Accuracy }=(\mathrm{TP}+\mathrm{TN}) / \mathrm{N}=(24+10) / 35=97 \%
\end{aligned}
$$

\section{Conclusion}

The image processing methods are utilized to identify breast cancer in mammogram images using MATLAB, which needs careful processing to obtain an accurate diagnosis of the disease without error. The proposed approach with image enhancement by the median filter for noise remove, the features of mammograms images have been extracted using Watershed Segmentation, and then extract tumor by Morphological operation (close and open operation). This proposed algorithm helps to know the stage of the tumor from mammogram images in emergencies and provide a preliminary result for the user and not to make a final decision on the existence of cancerous variations in an image. The system showed better performance when compared to the current algorithms and speed of implementation, so it is very useful for medical personnel in the discovery of the tumor. The proposed approach similarly obtained breast tumor diagnosis in all medical image types and the results obtained are good for the measurements used, for this reason, breast cancer has decreased due to better diagnostic facilities and effective treatments. 


\section{$7 \quad$ References}

[1] Saira Charan, Muham mad Jaleed Khan, Khurram Khurshid, Breast Cancer Detection in Mammograms using Convolutional Neural Network, international conference on computing, mathematics and engineering technologies, 2018. https://doi.org/10.1109/icomet. $\underline{2018.8346384}$

[2] N. Lanisa., Ng S. Cheok, Lai K. Wee, Color Morphology and Segmentation of the Breast Thermography Image, conference on biomedical engineering and science, IEEE, December 2014, Miri, Sarawak, Malaysia. https://doi.org/10.1109/iecbes.2014.7047614

[3] N. S. Ali, N. F. Soliman, M. Abdallah, F. E. Abd El-Samie, An algorithm for Pre-processing and Segmentation of Mammogram Images, 978-1-5090-3267-9/16, IEEE 2016. https://doi.org/10.1109/icces.2016.7821997

[4] M. R. Al-hadidi, A. Alarabeyyat, M. Alhanahnah, Breast Cancer Detection using K-nearest Neighbor Machine Learning Algorithm, international conference on developments in esystems engineering 9th IEEE, 2016. https://doi.org/10.1109/dese.2016.8

[5] Angayarkanni.N, Kumar.D and Arunachalam.G, The Application of Image Processing Techniques for Detection and Classification of Cancerous Tissue in Digital Mammograms, Sci\&Res, Volume. 8, 2016.

[6] A. Chattaraj, A.Das, M. Bhattacharya, Mammographic Image Segmentation by Marker Controlled Algorithm, international conference on bioinformatics and biomedicine, IEEE 2017. https://doi.org/10.1109/bibm.2017.8217793

[7] N. N. Shah, T. V. Ratanpara, C.K.Bhensdadia, Early Breast Cancer Tumor Detection on Mammogram Images, IJCA Journal, Vol.87, Number. 14, February 2014. https://doi.org/ $\underline{10.5120 / 15275-3871}$

[8] S. M. Badaway, A. A. Hefnawy, H. E. Zidan, Breast Cancer Detection with Mammogram Segmentation: A Qualitative Study, IJACSA international journal of advanced computer science and application, 2017.

[9] P Mohamed Shakeel, Amr Tolba, Zafer Al-Makhadmeh, Mustafa Musa Jaber, Automatic detection of lung cancer form biomedical data set using discrete Adaboost optimization ensemble learning generalized neural networks, neural computing and applications, springer London, 2019. https://doi.org/10.1007/s00521-018-03972-2

[10] Sura R. Shareef, Breast Cancer Detection Based on Watershed Transformation, IJCSI journal, Volume.11, Number.1, January 2014.

[11] J. Sharma, and S. Sharma, Mammogram Image Segmentation Using Watershed, international journal of information technology and knowledge management, volume.4, Number. $2,2011$.

[12] Wen-feng kuo, Chi-yuan lin and Wei-yen hsu, Medical Image Segmentation Using the Combination of Watershed and Fcm Clustering Algorithms, international journal of innovative computing, information and control, September 2011.

[13] Abdalrada, Ahmad Shaker, Omar Hashim Yahya, Abdul Hadi M. Alaidi, Nasser Ali Hussein, Haider TH Alrikabi, and Tahsien Al-Quraishi Al-Quraishi. "A Predictive model for liver disease progression based on logistic regression algorithm." Periodicals of Engineering and Natural Sciences 7, no. 3 (2019): 1255-1264. https://doi.org/10.21533/pen.v7i3. $\underline{667}$

[14] Ravi S, A M Khan, Morphological Operations for Image Processing: Understanding and its Applications, CTCEEC conference proceedings, 2017.

[15] Alrikabi, Haider TH, et al. "Analysis the Efficient Energy Prediction for 5G Wireless Communication Technologies." International Journal of Emerging Technologies in Learning (iJET) 14.08 (2019): 23-37. https://doi.org/10.3991/ijet.v14i08.10485 
[16] M R. Bai, V V. Krishna and J. Sree Devi, A new Morphological Approach for Noise Removal cum Edge Detection, IJCSI international journal of computer science issues, Vol. 7, Issue 6, November 2010.

[17] H. Tulsani, S. Saxena and N. Yaday, Segmentation Using Morphological Watershed Transformation for Counting Blood Cells, international journal of computer applications and information technology, volume.2, 2013.

\section{Authors}

Sarah Faris Ameer - Ministry of Justice, Notary public office, Department of Computer science, Dijlah University CollegeEmail: sarah.faris@duc.edu.iq

The number of articles in national databases: 1 . The number of articles in international databases: 2 .

Zinah Tareq Nayyef - Department of Computer science, Dijlah University College, Email: zeena.tariq@duc.edu.iq. The number of articles in international databases: 2

Zena Hussain Fahad - Department of Computer science, Dijlah University College, Email: zena.hussain@duc.edu.iq. The number of articles in international databases: 2.

Ibtihal Razaq Niama ALRubee - Department of Electrical Engineering, Wasit University, Al Kut, Wasit, Iraq

Article submitted 2020-01-03. Resubmitted 2020-02-25. Final acceptance 2020-02-27. Final version published as submitted by the authors. 